\title{
Anabases
}

ANABASES Traditions et réceptions de l'Antiquité

19 | 2014

Varia

\section{Sotera FORNARO \& Daniela SUMMA (a cura di), Eidolon. Saggi sulla tradizione classica}

\section{Daniela Bonanno}

\section{OpenEdition}

Journals

Édition électronique

URL : http://journals.openedition.org/anabases/4728

DOI : $10.4000 /$ anabases. 4728

ISSN : 2256-9421

\section{Éditeur}

E.R.A.S.M.E.

\section{Édition imprimée}

Date de publication : 1 avril 2014

Pagination : 341-343

ISSN : 1774-4296

\section{Référence électronique}

Daniela Bonanno, «Sotera fornaro \& Daniela summa (a cura di), Eidolon. Saggi sulla tradizione classica », Anabases [En ligne], 19 | 2014, mis en ligne le 01 avril 2014, consulté le 22 septembre 2020. URL: http://journals.openedition.org/anabases/4728 ; DOI : https://doi.org/10.4000/anabases.4728

Ce document a été généré automatiquement le 22 septembre 2020.

(c) Anabases 


\title{
Sotera FORNARO \& Daniela SUMMA (a cura di), Eidolon. Saggi sulla tradizione classica
}

\author{
Daniela Bonanno
}

\section{RÉFÉRENCE}

Sotera FORNARO \& Daniela SUMMA (a cura di), Eidolon. Saggi sulla tradizione classica, Bari, Pagina, 2013, $151 \mathrm{p}$.

15 euros/ ISBN 978-88-7470-281-7

1 Eidolon è il titolo di questa piccola raccolta di contributi sulla Tradizione classica, esito di un incontro tenutosi a Freiburg nel febbraio del 2013.

2 Come spiega S. Fornaro, nella premessa, il volume è articolato in tre sezioni : la prima dedicata alla tradizione letteraria, la seconda alla storia degli studi e la terza al dibattito novecentesco sulla mitologia. Apre la prima sezione il contributo di M. Castellari (Taboris letzter Brecht: Antigone-Variationen 2006, p. 11-22) che analizza la messa in scena dell'Antigone di Bertolt Brecht, rimaneggiamento della traduzione di Hölderlin della tragedia sofoclea. L'allestimento curato dal regista ungherese George Tabori cade nel cinquantesimo anniversario della morte del drammaturgo tedesco. Castellari indaga i perché della scelta dell'Antigone per l'occasione e li rintraccia nella lunga frequentazione del regista con l'opera del drammaturgo tedesco. Meno decisiva sarebbe invece la relazione con l'antico. Riproponendo la lettura politica dell'Antigone di Brecht, Tabori punta l'attenzione sul potenziale distruttivo che un regime totalitario esercita sulle nuove generazioni. Una nuova Antigone allunga dunque così il catalogo di quelle esaminate da George Steiner, il cui saggio Antigones (1984) avrebbe, forse, meritato una menzione in bibliografia.

3 Al Prometeo di Goethe dedica la sua attenzione S. Fornaro (Le forme dell'amore nel frammento drammatico Prometeo di Goethe, p. 23-34), un'opera giovanile articolata in 
quattro dialoghi morali privi di azione da cui emerge una rappresentazione del Titano inedita rispetto a quella della tradizione classica. È l'immagine di un Prometeo dimidiato tra arte e amore. Nei versi il legame con la tradizione classica è garantito dalla simbiosi con la Musa, espressione di una forza divina che dà voce al poeta e gli conferisce, secondo l'ideale omerico ed esiodeo, conoscenza e una capacità di raccontare che ha del sovraumano. La tradizione classica è poi, però, relegata sullo sfondo dai tratti erotici assunti da questa relazione simbiotica e dal velo di malinconia di cui essa si copre.

Chiude questa prima sezione il saggio di M. Marino, Dichtung, Philosophie und Religion:

Herders erstes Exzerpt aus Lukrez' De rerum natura und dessen Wirkung auf Herders Philosophie der Geschichte der Menschheit, p. 35-61. Un manoscritto di Johann Gottfried Herder, che contiene la sua prima documentata lettura del De rerum natura di Lucrezio, offre il destro per esplorare il rapporto tra il filosofo tedesco e il poeta latino. Dopo un breve status quaestionis, l'A. passa all'esame degli appunti di Herder da cui emerge in trasparenza, da un lato, il contributo dell'etica epicurea che, per il tramite di Lucrezio, giunge fino ad Herder; dall'altro un'approfondita riflessione da parte del filosofo tedesco sul ruolo della poesia, quale strumento per avvicinarsi alla conoscenza del mondo e di sé, che non nega tuttavia l'apporto della scienza moderna alla costruzione del sapere umano.

5 La sezione dedicata alla storia degli studi si apre con il contributo di C. Bonnet, L'époque solsticiale de l'histoire ancienne: Carthage et Rome au coeur des dynamiques Est-Ouest dans la Römische Geschichte de Theodor Mommsen, p.63-82. Al centro di questa indagine è il ruolo delle guerre puniche all'interno della Römische Geschichte di Theodor Mommsen. «Mostro sacro dell'Altertumswissenschaft tedesca » (p. 64), come lo definisce l'A. stessa, e infaticabile editore del Corpus Inscriptionum Latinarum, avviato sotto l'egida dell' Accademia delle Scienze di Berlino, Mommsen ottiene proprio grazie a quest'opera il premio Nobel per la Letteratura nel 1902. Chiaro sin dal principio è il progetto storiografico che la sostiene, il cui cardine è programmaticamente individuato nel processo di unificazione delle stirpi italiche in un solo stato. Le interferenze tra passato e presente sono evidenti e lasciano leggere in controluce l'appoggio iniziale dato dallo studioso al piano di unificazione tedesca a guida prussiana. All'interno di questo progetto, Cartagine e le guerre puniche giocano un ruolo chiave soprattutto in ragione del peso che i Fenici esercitarono all'interno degli equilibri geopolitici tra Oriente e Occidente. La posizione assunta da Roma sullo scacchiere internazionale mediterraneo, dopo la distruzione di Cartagine, e la dilatazione del suo spazio d'azione, la trascineranno in una deriva espansionistica che ne trasformerà radicalmente il volto, dando avvio a un processo di decadenza i cui esiti saranno evidenti in età imperiale.

6 L'Accademia delle Scienze di Berlino torna nel contributo di Daniela Summa (Il progetto Inscriptiones Graecae tra passato e presente. L'esempio Cipro (IG XV), p. 83-106), un percorso appassionante, arricchito da un ampio corredo di documenti inediti, sulle vicende storiche e politiche che, per più di un secolo, hanno ostacolato la realizzazione del corpus delle iscrizioni di Cipro. Dopo una breve introduzione sulla storia del progetto delle Inscriptiones Graecae presso la Berlin-Brandenburgische Akademie der Wissenschaften, l'A. illustra le circostanze che portarono, all'inizio del Novecento, alla « diaspora » delle antichità cipriote in vari musei del mondo, tra cui spicca l'attività del console Luigi Palma di Cesnola, legata a celebri, quanto paradossali, falsificazioni archeologiche. Lo scoppio delle due guerre mondiali e la divisione della Germania 
impedirono poi il rilancio dell'edizione del corpus epigrafico cipriota. Dopo la caduta del muro, il progetto è stato ripreso con qualche modifica rispetto al piano originario. Benché esso sia ora rallentato dalla divisione politica di Cipro che rende difficilmente accessibili alcuni siti, l'équipe di ricerca oggi incaricata della realizzazione del corpus, di cui l'A. fa parte, sta portando avanti, nel frattempo, lo studio delle iscrizioni sparse nelle varie collezioni, con l'obiettivo di portare finalmente a termine l'opera.

La terza sezione è costituita da due saggi che analizzano la funzione politica, sociale e letteraria del mito nel dibattito novecentesco tra Germania e Italia. Carlotta Santini ( $L a$ seduzione del mito. Furio Jesi, Thomas Mann e Karoly Kerényi, p. 107-123) prende le mosse dall'incontro epistolare tra Károly Kerényi e lo scrittore Thomas Mann che si riproduce, trent'anni dopo, tra lo storico delle religioni italiano Furio Jesi e lo stesso Kerényi. Al centro dei due epistolari vi è proprio il valore politico, religioso e sociale del mito e il processo di « secolarizzazione » cui esso era andato incontro, amplificato dall'ideologia nazista. Kerényi riconosceva al mito una funzione epistemologica e gnoseologica e trovava a questo proposito una conferma nell'opera di Mann, al quale - occorre aggiungere - lo legava anche una profonda amicizia basata su una consuetudine di rapporti personali. Lo scrittore tedesco, dal canto suo, individuava nel mito un potenziale demoniaco, testimoniato dagli abusi del suo tempo. Al dibattito tra Mann e Kerényi si aggiunge, molti anni dopo, la voce di Furio Jesi, espressione di una generazione di intellettuali che, pur non avendo vissuto gli orrori della guerra, avverte l'esigenza di un momento di riflessione morale e politica su di essa. Oggetto della critica di Jesi è ancora una volta il mito, inteso sia come « immagini » e « concezioni » sia come « atmosfere spirituali » che tanto la cultura tedesca quanto l'ideologia nazista avevano in comune : « immagini di morte » che, nonostante la difesa a oltranza di Kerényi, egli riteneva che Mann avesse accolto nella sua opera. In questo quadro, avrebbe forse meritato un cenno, la distinzione tra «mito genuino" e "mito tecnicizzato" che Kerényi e Jesi portarono avanti e che sembra appena adombrata nella conclusione.

Chiude il volume il saggio di Eleonora Cavallini (Pavese tra gli dèi: Calvino primo commentatore dei Dialoghi con Leucò, p. 125-144), l'unico dedicato al contesto italiano. L'A. ricostruisce, attraverso il commento di Italo Calvino ai Dialoghi con Leucò di Cesare Pavese, l'interesse dello scrittore italiano per il mito e la sua capacità di leggerlo, non solo da filologo, ma anche con gli occhi del moderno etnologo. Un interesse confermato dall'attenzione di Pavese per gli studi storico-religiosi italiani rappresentati da A. Brelich e R. Pettazzoni, dalla collaborazione editoriale con E. De Martino e dall'impulso dato a grandi imprese editoriali come la traduzione dell'lliade di R. Calzecchi Onesti per Einaudi. Il giudizio pur lusinghiero espresso da Calvino lascia intravvedere i fili di un dibattito che andava liquidando la funzione del mito come modalità espressiva, ormai vista con sospetto per gli abusi cui era stata sottoposta dei regimi totalitari e considerata inadeguata alla realtà contemporanea.

Un indice dei nomi correda questo volume nei cui contributi risuona l'eco rifratta di una feconda tradizione classica e delle possibili forme attraverso cui, nel panorama culturale tedesco, per lo più, si prestava a essere riplasmata, reinventata, quando non strumentalizzata, per rispondere a progetti culturali ben precisi o a contingenze temporali. 


\section{AUTEURS}

\section{DANIELA BONANNO}

Università degli Studi di Palermo

daniela.bonanno@unipa.it 